\title{
Le « dire » dusilence : les ponctuations émotionnelles et cognitives des interactions
}

Arshad Malik

(2) OpenEdition

Journals

Édition électronique

URL : https://journals.openedition.org/communicationorganisation/2413

DOI : 10.4000/communicationorganisation.2413

ISSN : 1775-3546

\section{Éditeur}

Presses universitaires de Bordeaux

Édition imprimée

Date de publication : 1 novembre 2000

ISSN : 1168-5549

Référence électronique

Arshad Malik, "Le «dire » dusilence : les ponctuations émotionnelles et cognitives des interactions », Communication et organisation [En ligne], 18 | 2000, mis en ligne le 27 mars 2012, consulté le 05 août 2021. URL : http://journals.openedition.org/communicationorganisation/2413 ; DOI : https://doi.org/ 10.4000/communicationorganisation.2413

Ce document a été généré automatiquement le 5 août 2021.

(C) Presses universitaires de Bordeaux 


\title{
Le « dire » dusilence : les ponctuations émotionnelles et cognitives des interactions
}

\author{
Arshad Malik
}

1 Le silence est notre conscience de l'absence sonore. Ainsi, le sens du silence est en fonction de son rapport avec notre sensorialité-perception. La réalité globale et complexe du silence se présente sous forme d'une expérience, bio-physiologique, psychique et culturelle à la fois.

\section{Du silence}

2 En premier lieu, le silence vu uniquement comme expérience bio-neurophysiologique est une condition de survie pour l'organisme. C'est un besoin ontologique vital. Un besoin pour se protéger des agressions sonores du milieu. C'est un silence que le rythme biologique impose aux vivants, pour que l'organisme se repose. C'est la nuit contre le jour. Ce silence de repos et de calme, dit, qu'il voudrait se maintenir. Ainsi, l'existence de ce silence réside, dans la neutralisation-inhibition de non-silence. Le silence de repos invite au silence, inspire au silence, comme une condition de son respect et de sa révérence. C'est le panneau «silence » dans la proximité d'un hôpital, qui dit que l'organisme, souffrant, malade, fatigué, fragile, mort ou mourant, aspire au silence. Un tel silence parle pour conserver sa durée. Ainsi le silence comme un besoin vital se comporte comme un devoir de silence.

\section{Le silence}

3 Le silence en tant que réalité phénoménale est le silence des éléments : c'est dans la façon par laquelle l'homme perçoit et sent mystérieuse et inerte-muette l'existence des phénomènes naturels. C'est à la fois le silence de l'espace statique-immobile et l'espace mobile : le ciel, le mouvement des astres dans le ciel, la montagne, la forêt, le désert, les 
ruines, le vide, l'océan, etc. Nous savons que la nature n'est jamais complètement silencieuse, mais notre conscience du silence devant l'étendue des phénomènes naturels est au fond une expérience émotionnelle et esthétique. C'est l'état de lassitude devant l'animation familière, et la quête imaginaire et poétique de «luxe, calme et volupté ». Le silence comme expérience émotionnelle et esthétique a des dimensions à la fois épistémiques, mystiques et artistiques. Le silence de la nature est au fond le silence du réel, c'est un défi, pour connaître, c'est le mystère de l'inconnu. C'est toujours l'inconnu et l'inconnaissable qui constituent l'essence du sacré, du divin, du totémique. La sacralisation des phénomènes les entoure de la révérence, par conséquent du silence.

\section{Des silences}

4 Outre le constat biologique et phénoménologique du silence, l'essentiel du rapport humain au silence réside au sein de la dynamique relationnelle, interactive, communicative. Dans ce contexte, d'un côté, sans le silence, la parole, la communication orale n'a pas de sens, de l'autre, le silence est une entité signifiante autonome et complexe. On peut même dire que le silence a un réel statut discursif: puisque selon la conception classique « tout est langage » (F. Dolto), ou encore la thèse chère à l'école de Palo Alto " on ne peut pas ne pas communiquer ", tout comportement a une valeur de message, par conséquent une fonction communicative. Ainsi, ayant son inscription au sein de la complexité des comportements humains, l'analyse du silence exige au moins une triple approche conceptuelle: linguistique, psychologique, anthropologique. En effet, considérant le silence comme un langage à part entière, il peut être pleinement saisi à travers le schéma tridimensionnel introduit par le sémioticien Charles W. Morris, à savoir: syntaxe, sémantique et pragmatique. À la différence d'autres systèmes sémiotiques, le silence serait la réalité symbolique absolue, puisque son contenu symbolique ne peut pas être matérialisé. En tant que signe ponctuant l'espace sonore, sa matérialité syntaxique n'est pas autre que la relative conscience de non-audition. Ainsi, la syntaxe du silence est une entité temporelle, à savoir une durée donnée de silence. Mais une autre manière d'établir la syntaxe du silence serait de constituer ses typologies. Ces typologies ne peuvent pas être fonction de la durée d'un silence, mais plutôt du contexte interactif et du type de relation en jeu. Par exemple, un silence: de menace, de peur, de haine, de rejet, de communion, de révérence, d'écoute, de réflexion, de timidité, d'inhibition, etc. Selon cette approche, un type donné de silence serait aussi la forme ou le contenu syntaxique de ce silence.

5 Si dans le cadre des analyses sémiotiques ordinaires, il est possible d'étudier la syntaxe, la sémantique et la pragmatique séparément, le silence comme état communicatif s'impose dans son unité complexe tripolaire. Ainsi, lorsque le silence parle (syntaxe) il véhicule en même temps le sens (sémantique) et l'effet (pragmatique). Par ailleurs, cette unité significative du silence demeure une réalité libre et fluctuante. Dans le contexte de la communication verbale, l'interdépendance entre la syntaxe et la sémantique est la condition même de la communication, puisque la dimension arbitraire et conventionnelle de la syntaxe est là, pour contenir le chaos du sens, même si dans l'absolu le sens langagier déborde toujours son sens norme. Par contre, dans le domaine non-verbal, et surtout le non-verbal du silence, le rapport au sens libre et intime du sujet devient un élément fort dans le rapport de force interactif. Alors les 
stratégies de manipulations-exploitations entre les parties impliquées se font par le biais d'un jeu de sens ; ce jeu est d'autant plus subtil que le cadre contenant le sens de manière objective (la syntaxe) est fluctuant, mobile, voir absent. Malgré cette difficulté pour la saisie du sens exact du silence, nombre des comportements humains (conscients, inconscients), sa typologie sémantique serait sans doute inhérente à la logique pragmatique des enjeux interactifs. Ainsi repère-t-on deux grands registres des silences: les silences comme symptômes et les silences comme stratégies d'actions communicatives décrivant les conduites défensives/offensives. Parallèlement bien entendu, à l'immense variétés des silences, vécus individuellement, interactivement ou collectivement.

6 Les silences comme symptômes sont le reflet de la complexe et mystérieuse réalité psychique consciente-inconsciente de l'homme. Ces états symptomatiques vont des pathologies psychiques graves jusqu'aux perturbations-troubles passagers vécus quotidiennement. Le silence à travers l'état de mutisme et de recroquevillement chez une personne autiste est une étrange et troublante communication dans la noncommunication qui représente un terrible défi pour "L'herméneutique thérapeutique ». Si, tout comportement humain est sujet au regard interprétatif de l'autre, les conduites symptomatiques inspirent encore plus la saisie du sens sousjacent. On dit bien : « il ne dit rien, il est de plus en plus renfermé, serait-il déprimé ?». On observe encore du silence lié à la timidité, l'inhibition, les changements d'humeur. $\mathrm{Au}$ sein de l'espace affectif-émotionnel intime (couple etc.) le silence du «faire la gueule » décrit la tension préalable de la crise (explosion). Après le passage de tonnerre et de l'orage, règne le silence de relâchement, de vide, de décharge ou encore les silences de mal-être, de chagrin, de tristesse, de solitude affective etc. D'une façon plus générale, la problématique du silence comme symptôme souligne que le psychisme dans sa construction même constitue les zones du silence. L'inconscient est la partie cachée et silencieuse du sujet. Le processus subtil d'inhibition et de refoulement fait passer sous silence ce que le sujet ne peut, et ne veut pas voir en face. Aussi, l'extériorisation du refoulé se fait sous des formes de mouvements symptomatiques silencieux, tels le silence des rêves, le silence sous-jacent au symbolisme mythiquerituel collectif, ou encore le silence derrière la manifestation psychosomatique. Dans le silence psychosomatique, le non-dit, le non-accepté, et le non-résolu sortent par le corps. Mais les plus spectaculaires manifestations du refoulé (la part silencieuse des sujets) se réalisent au sein des espaces culturels globaux. Ce sont les fêtes, cérémonies, rites, rituels autour du sacré (chamanisme, sorcellerie, initiation etc.) et du sexuel (carnaval etc.) où les angoisses (de mort et de castration) passées sous silence sont mises en scène et canalisées. Ces mises en scène déguisées du refoulé, du non-dit, constituent l'image d'un silence collectif en fête. La manifestation, le vécu de ces fêtes et des cérémonies ne sont, ni la fin du silence, ni forcément l'accès à la zone silencieuse chez les sujets, puisque la régulation psychique collective est sensée se faire sans lever le voile du silence. Le surmoi, le normatif collectif, l'institutionnel, sont là pour imposer et pérenniser ce silence qui fait partie intégrante du psychisme.

7 Le silence comme stratégie interactive s'exprime pour l'essentiel par le secret et la dissimulation. Le rapport entre l'homme et la dissimulation est un rapport vital, puisque c'est la condition même de son être, de sa liberté et de sa normalité psychique. Depuis la nuit des temps et en commun avec d'autres espèces des vivants, les stratégies de défense contre les prédateurs et les ennemis sont basées sur la dissimulation qui va du déguisement morphologique du caméléon au secret défense, au secret d'état, au 
secret du crime, jusqu'aux pratiques de détection et d'espionnage électroniques informatisées les plus actuelles. Le silence est autant offensif que défensif. Lorsqu'un prédateur ou un ennemi attaque, il se cache et il contrôle au maximum le «bruit ». Par ailleurs au plan symbolique des interactions humaines, un silence offensif et violent désarme l'autre, et ceci en le négligeant, l'excluant, l'excommuniant mais aussi en l'«attaquant par-derrière ». Ainsi, la finalité globale du silence serait de pouvoir se protéger physiquement et psychiquement de l'emprise de l'autre. À travers les socialisations successives, l'individu humain avance vers la construction subjective normale et vers l'adaptation sociale, et ceci grâce à deux expériences vitales : lorsqu'il dépasse la confrontation frontale et. lorsqu'il apprend à gérer, entre se taire et dire. Autrement dit, il apprend (explicitement et tacitement), comment dire, où dire, à qui dire, ainsi que, où se taire, comment se taire, pourquoi se taire etc. La rencontre inédite entre les individus inconnus est un état de danger, de menace pour chaque partie. Mais en réalité, la menace et le danger sont d'autant plus grands que la rencontre est sous le signe du silence. Finalement, c'est le silence qui est menaçant. Ainsi, ce silence communique de la panique, de l'anxiété, de la peur. C'est la phase de communication frontale, explosive, brute, archaïque, non ritualisée, non socialisée, voire non symbolisée. Dans cette situation, le premier message que le silence véhicule, c'est son insupportabilité et, à terme, sa fin. Cet état non-verbal d'interaction a besoin d'être dompté, apprivoisé, voir exorcisé. Décoder un silence, c'est pouvoir lui donner du sens. Le silence dans les interactions inédites frontales empêche l'émergence du sens. Le silence non décodable des interactions a pouvoir de dé symbolisation, ainsi que la régression vers des états pulsionnels. C'est ce pouvoir décontenançant et déroutant du silence qui rend fou. Le fait «silence » non socialisé est souvent socialisé par une autre modalité non-verbale universelle, à savoir le sourire. C'est ainsi que le sourire humanise, ritualise l'interaction silencieuse. Aussi, nous constatons que la plupart des rites, rituels, d'interaction non-verbales (sourires, poignées de mains, embrassades etc.) et verbales (salutations etc.) sont des réponses au message silence. Outre la dimension menaçante du silence (interaction non ritualisée, non symbolisée) des stratégies interactives, selon la logique du rapport de force, le silence peut être utilisé comme une violence perverse (patente ou latente) sous forme d'état de mépris de l'autre. Au sein des contextes institutionnels et organisationnels, les personnes hiérarchiquement dominantes, si elles utilisent souvent la stratégie de rétention (dissimulation) des informations, elles pratiquent aussi le dosage calculé entre la parole et le silence. Si le silence glacial d'un chef peut faire trembler tout subordonné, le silence ciblé et répétitif d'un supérieur hiérarchique agit comme une violence symbolique, voire comme un « harcèlement moral », en cultivant un sentiment de culpabilité, de doute, d'inefficacité chez le subordonné. Parfois, une telle expérience vécue par un subordonné peut créer à long terme des perturbations psychologiques (anxiété, stress, etc.) et sociales (retraite, isolement, etc.). Afficher, imposer, le silence devant quelqu'un, en tenue symbolique, c'est acter sa mise à mort. La pratique traditionnelle de l'excommunions peut tuer (symboliquement) le déviant par le silence collectif, ferme, et unanime. C'est un contexte ultime au " quand non-dire, c'est faire ${ }^{1}$ "; ainsi le silence se comporte comme un véritable acte de langage. L'excommunions n'est pas à confondre avec la « loi de silence ». En rapport avec la loi, le silence comme régulateur social et culturel, agit des deux manières suivantes. Lorsque l'espace culturel lui-même passe sous silence le normal d'une façon telle que l'illégal anormal, devient normal, et lorsque le principe de vengeance systématique et généralisé instaure l'altérité pulsionnelle, alors la loi se tait 
pour devenir la « Loi du silence ». Lorsque la mort plane partout, le voile de silence sert d'une relative protection et tente de contenir la violence qui est à fleur de peau.

Un autre mode interactif où le silence s'exprime avec ses pleines conséquences, est la situation de "communication paradoxale». L'injonction qui est latente au message paradoxal peut véhiculer de multiples contraintes vers le récepteur. Ainsi l'injonction silencieuse n'est pas autre chose qu'un état de sens, qui a plus de pouvoir que le sens manifeste. De toute manière, le sens du silence est toujours porteur d'un poids supplémentaire d'imaginaire et de fantasmatique.

Parmi tout les silences associés à la réalité interactive de l'homme, le plus répandu cela va de soi, est le silence de l'écoute. C'est la condition même de la communication et de la compréhension. On peut observer une grande variété de silences d'écoute. Le silence du psychanalyste est une condition de l'écoute de l'analysant et c'est un élément essentiel du cadre analytique. Le silence souhaitable des élèves d'une classe est reconnu comme condition de réussite des apprentissages. Outre les conséquences émotionnelles très variables du silence sur les individus, la valeur du silence se mesure surtout en fonction de son lien avec la cognition. Si l'écoute silencieuse de l'autre met en scène la cognition interactive, au plan individuel, le silence est parfois la condition même de la pensée. Pour penser pleinement, il faut s'écouter. Ce rapport d'écoute silencieuse avec soi-même sous forme d'une double démarche, méta cognitive et métapsychique, permet au sujet d'écouter et de voir son état affectif et cognitif à la fois. Aussi en rapport avec la normalité psychique, pouvoir penser secrètement est la condition de la construction subjective et de la liberté. Lorsque l'individu est transparent à l'autre il n'est ni libre, ni normal.

Au delà de son ancrage psychique profond, notre confrontation quotidienne au silence oscille entre les multiples finalités pragmatiques qui peuvent aller du silence ludique et léger $\mathrm{du}$ « cache-cache » des enfants, au silence sadique qui « attend l'autre au virage ».

11 De manière globale on constate que le silence représente les deux extrémités des interactions humaines, à savoir communication danger et communication fusion/ communion. Ce double rapport au silence est une double démarche cognitivo-affective. liée à la compréhension-incompréhension et à la sécurité-insécurité. Si la rencontre frontale inédite est un moment d'incompréhension et d'insécurité, dans le silence de la communion règne la compréhension et la confiance.

\section{BIBLIOGRAPHIE}

HIRIGOYEN M. F., Le harcèlement moral : lu violence perverse au quotidien, Syros, Paris, 1998.

WATZLAWICK P., HEIMICK BEAVIN J., JACKSON D.. Une logique de la communication, Paris, Seuil, 1972.

SEARLE J.R., L'intentionnalité : essai de philosophie des états mentaux. Minuit, Paris, 1985.

OLIVENSTEIN C, Le non-dit des émotions. Odile Jacob. Paris 1988. 
NOTES

1. Nous taisons ici référence au titre du livre de J.L. Austin, Quand dire c'est luire. Seuil, Paris, 1970

AUTEUR

ARSHAD MALIK

IUFM Toulouse 